\title{
(2) OPEN ACCESS \\ Case for a new corticosteroid treatment trial in optic neuritis: review of updated evidence
}

\author{
Axel Petzold (D) , ${ }^{1,2}$ Tasanee Braithwaite, ${ }^{3}$ Bob W van Oosten, ${ }^{4}$ Lisanne Balk, ${ }^{5}$ \\ Elena H Martinez-Lapiscina (1) , ${ }^{6}$ Russell Wheeler, ${ }^{7}$ Nils Wiegerinck, ${ }^{8}$ \\ Christiaan Waters, ${ }^{9}$ Gordon T Plant $^{10}$
}

For numbered affiliations see end of article.

Correspondence to Dr Axel Petzold, Amsterdam UMC, Amsterdam, NoordHolland, Netherlands; a. petzold@ucl.ac.uk

Received 16 July 2019 Revised 20 September 2019 Accepted 16 October 2019 Published Online First 18 November 2019

Check for updates

(c) Author(s) (or their employer(s)) 2020. Re-use permitted under CC BY-NC. No commercial re-use. See rights and permissions. Published by BMJ.

To cite: Petzold A,

Braithwaite T, van Oosten

BW, et al. J Neurol Neurosurg Psychiatry 2020:91:9-14.

\section{INTRODUCTION}

Optic neuritis $(\mathrm{ON})$ is a condition that causes loss of vision. Most frequently, ON affects one eye, but occasionally, simultaneous bilateral loss of vision occurs. Typically, a variable degree of spontaneous recovery takes place within about 3 months and can continue for up to 1 year. There are a number of triggers for $\mathrm{ON}$, ranging from postvaccination episodes to any type of inflammation and specific autoimmune conditions such as multiple sclerosis (MS), neuromyelitis optica (NMO), and myelin oligodendrocyte glycoprotein (MOG) ON among $^{12}$ others. In about $5 \%$ of patients, there is risk of severe permanent loss of vision and blindness. A major challenge is that it is not possible to know the subtype of $\mathrm{ON}$ at presentation. While certain demographic features, symptoms and clinical signs are suggestive, more definitive results from blood tests and neuroimaging can take days or weeks to obtain. Furthermore, negative findings in the blood tests do not rule out non-multiple sclerosis-associated optic neuritis (MSON).

The clinical management of patients who suffer from MSON has been profoundly influenced by the 1992 US Optic Neuritis Treatment Trial (ONTT). ${ }^{3}$ This trial recommended intravenous corticosteroids, not to improve the final outcome but to speed up visual recovery. ${ }^{4}$ There were, however, limitations to the ONTT protocol, which may have influenced the findings. ${ }^{5}$ First, the ONTT used a vague definition of 'symptom onset'. This is relevant because loss of vision (used in the ONTT) is frequently preceded by several days of pain from inflammation. ${ }^{6}$ Second, there was delayed treatment initiation, at a mean of $5.0 \pm 1.6$ days, which was timed from the visual loss but not from the onset of pain. Third, by contemporary standards, the trial primary outcome measure of high-contrast visual acuity (HCVA) was relatively crude. Fourth, the ONTT recruited a heterogeneous cohort of ON types, including a minority of MOG antibodypositive cases, ${ }^{7}$ who may well behave differently from MSON and be corticosteroid sensitive and even corticosteroid dependent. There were no aquaporin 4 (AQP4) seropositive cases in the ONTT. Furthermore, the ONTT did not include any pharmacokinetic data. It was thus not possible for investigators to comment on blood levels, resulting in an inability to propose a plausible biological explanation for as to why oral versus intravenous corticosteroids should be harmful compared with intravenous corticosteroids. ${ }^{89}$
Given these limitations, h it is not surprising that more recent non-inferiority trials have refuted the finding that intravenous corticosteroid treatment is superior to oral administration. ${ }^{10-12}$

The ONTT leaves unanswered at least two vital questions. If steroids are given within some 'critical window' of symptom onset, are they efficacious, in certain types of ON, or even in patients with demyelinating ON? Also if patients with $\mathrm{ON}$ of uncertain aetiology at presentation are given steroids promptly (eg, within 48 hours), can blindness be averted? Persisting uncertainty from this landmark trial has resulted in controversy and ongoing practice variation internationally, and the stakes are high. In this opinion piece, a case is made on the need for a new corticosteroid versus placebo treatment trial in $\mathrm{ON}$.

\section{NOVEL DIAGNOSTIC GROUPINGS}

Over the past 25 years, new developments have changed our understanding of the clinical spectrum and causes, the diagnostic workup and the impact of ON on patients. While testing for just syphilis, glucose and antinuclear antibodies was sufficient in $1992,{ }^{3}$ contemporary diagnostic workup includes testing for autoantibodies, such as those to AQP4 and MOG. ${ }^{6671313}$ It is now possible to subgroup patients with $\mathrm{ON}$ with greater precision in relation to aetiology, in order to explore differences in prognosis by subgroup. Table 1 summarises tests that should be considered for a new ONTT.

\section{NOVEL OUTCOME MEASURES}

Clinical measures of functional vision, such as HCVA, remain vitally important. A series of subgroup analyses of the ONTT have conclusively demonstrated that $>70 \%$ of patients with MSON recovered their HCVA to $\geq 6 / 6$ Snellen equivalent (US notation 20/20) within 1 year and essentially maintained this level for the next 14 years. ${ }^{14}$ However, there has arguably been an overdependence on visual acuity, a readily measurable and physician-favoured outcome metric, which may fail to capture important loss of visual function for the patient.

Validated, quantitative tests are now available to test low-contrast visual acuity (LCVA), including the Mars contrast sensitivity chart, and colour vision. ${ }^{15}{ }^{16}$ A seven-point change on a 2.5\% LCVA Sloane letter chart is considered as clinically meaningful. ${ }^{15}$ Highly accurate quantification of acquired 
Table 1 Summary of tests to be considered for a contemporary state-of-the art Optic Neuritis Treatment Trial

\begin{tabular}{|c|c|c|c|c|}
\hline Clinical & Patient reported & Imaging & Laboratory & Other \\
\hline HCVA & QoL & Funduscopy & MOG* & ERG + \\
\hline LCVA & PROMs & OCT & AQP4* & VEP† \\
\hline Colour vision & Service requirements & OCTA§ & ANA & Perimetry \\
\hline Pulfrich & Ease of service access & FAFt & Neurofilamentף & Pharmacokinetics \\
\hline RAPD** & Satisfaction & MRItt & Sample storage & \\
\hline
\end{tabular}

We strongly recommend storage of research blood samples as new biomarkers continue to be discovered.

${ }^{*}$ Cell-based assays recommended for MOG and AQP4.

tOn clinical grounds, in selected cases, FAF/ERG/VEP will be very helpful. We do not think this needs to be done routinely in every patient.

$\ddagger$ We recommend a macular volume scan of sufficient quality to permit layer segmentation and recognition of MMO and a peripapillary ring scan. Adherence to validated OCT quality control criteria and reporting guidelines is recommended. ${ }^{22}{ }^{61}$ There should be a central reading centre. ${ }^{64}$

$\S$ Same macular area as by OCT; optic disc only if device capable to reliably image.

१Neurofilament light and heavy chains should be quantified using a sensitive and validated immunoassay; batch analyses in single laboratory strongly recommended.

** Quantitative, pupillometric assessment of the RAPD. ${ }^{29}[68]$

††MRI sequences have been detailed in a consensus protocol. ${ }^{6}$ This protocol takes $\sim 10 \mathrm{~min}$ for the optic nerve, $\sim 20 \mathrm{~min}$ for the brain and, if required, an additional $\sim 15$ min for the spinal cord. Inclusion of research sequences such as diffusion tensor imaging will require more time.

ANA, antinuclear antibodies; AQP4, aquaporin 4; ERG, electroretinogram; FAF, fluorescein angiography; HCVA, high-contrast visual acuity; LCVA, low-contrast visual acuity; MOG, myelin oligodendrocyte; OCT, optical coherence tomography; OCTA, optical coherence tomography angiography; PROM, patient-reported outcome measure; QoL, quality of life; RAPD, relative afferent pupillary deficit; VEP, visual evoked potentials.

dyschromatopsia is possible using the CAD test, which has therefore become standard in the aviation industry. ${ }^{16}$ The quality of calibration of the CAD and validated age-related normative data to judge on clinical meaningfulness makes it a good choice for a multicentre trial setting. ${ }^{6}$ A resource-saving but not calibrated approach quantifies red desaturation and may be of interest for a single-centre trial with a limited budget. ${ }^{17}$

These may better capture the persisting decrement in low contrast or colour after $\mathrm{ON}$, which leaves patients struggling to function in low light conditions in spite of $6 / 6$ distance acuity. There has been a concentrated effort by the MS and neuroophthalmology community to highlight the value of LCVA, to capture the functionally relevant visual deficit that patients experience. ${ }^{1819}$ The LCVA overcomes the ceiling effect observed with HCVA measurements.

Technological advances are providing new quantitative measures that correlate with the patient-reported experience of their visual function following an episode of acute ON. Retinal axonal degeneration, which can be quantified with high precision by optical coherence tomography (OCT), has been established as a key pathological feature that is significantly correlated to visual acuity in ON. ${ }^{20}$ The degree of atrophy of the peripapillary retinal nerve fibre layer ( $p R N F L$ ) can be measured to within 1 micron. ${ }^{20} 21$ Similar considerations apply to the macular ganglion atnd inner plexiform cell layers (mGCIPLs). These highly robust outcome measures were not available at the time of the ONTT. ${ }^{20}$ A stepwise networking approach has contributed to rigorous OCT quality control criteria, which are now used in clinical trials. ${ }^{22-24}$ OCT has now been accepted as a validated outcome measure in 13/15 (86\%) of recent ON treatment trials ${ }^{25}$ because it demonstrably correlates with visual acuity. ${ }^{19} 26$ A 20\% intereye difference of OCT metrics has been proposed as a diagnostic for $\mathrm{ON},{ }^{6}$ but a series of recent validation studies showed that a $5 \%$ intereye difference gives a better sensitivity in a research setting. ${ }^{26-28}$ The intereye difference of the mGCIPL also correlates with quantification of the relative afferent pupillary deficit (RAPD) in ON. ${ }^{29}$ Taken together, OCT yields sensitive, objective and reproducible measures indicative of even small degrees of neurodegeneration.

Finally, recent years have seen greater awareness of the importance of the patient voice in ophthalmology. ${ }^{30}$ Patient-reported outcome measures (PROMs) are increasingly used in clinical trials to assess the impact of treatment from the patient perspective. ${ }^{31}$ Quality of life (QoL) is impacted both by the acute ON and its after effects and by its treatment. Finally, there is a differential economic impact of intravenous versus oral verus no therapy to consider, for patients and carers, and the health system.

Taken together, these three new dimensions of outcome measure permit a more sensitive capture of the impact of disease on patients and provide impetus for a new ONTT design. Involvement of patients and their advocates to prioritise the available outcome measures will be crucial.

\section{NOVEL PROGNOSTIC BIOMARKERS}

An important common shortcoming of earlier failed ON trials was the inability to model and predict a treatment response and long-term prognosis. Existing measures do not permit recognition of the extent of acute axonal damage at onset, which is unlikely to recover. Neither can electrophysiology (eg, visual evoked potentials), as conduction block and demyelination mask acute axonal damage. ${ }^{6}$ The only validated biomarker that can be used is the serum concentration of neurofilament $(\mathrm{Nf})$ proteins, which are validated body fluid biomarkers of neurodegeneration. $^{32} 33$

The Nf proteins are specific to the neuro-axonal compartment and are released into the adjacent body fluid compartment, the interstitial fluid, during acute damage. ${ }^{34}$ From the interstitial fluid, Nf diffuse into the cerebrospinal fluid and blood, where they can be readily quantified using immunoassays. ${ }^{33}$ Pioneering developmental work was followed by multicentre validation strategies, all of which helped to firmly establish this biomarker as an outcome measure, ${ }^{35-37}$ and we were first to show the prognostic value in acute ON. ${ }^{38}$ This has since been reproduced independently. ${ }^{33} 39$ From experimental models, we know that blood Nf levels rise within 30 min from injury. ${ }^{40}$ From human studies, we know that blood Nf levels persist for at least 3 months. ${ }^{41}{ }^{42}$ Blood Nf levels are an important predictor of treatment response, and their inclusion in a future ONTT would permit baseline stratification to identify those more or less likely to benefit from hyperacute treatment. They are also a secondary outcome measure for clinical trials. ${ }^{23} 3338$

\section{REGULATORY AUTHORITIES}

Regulatory authorities have an important role throughout the entire trial process, from early-phase studies to final drug licensing approval, and this is oftentimes overlooked. Our own observation, from interactions with the Food and Drug Administration (FDA) and the European Medicines Agency (EMA), is that there will be a requirement for the clinical research community to demonstrate the validity of novel outcome measures, 
especially including measures of anatomical structural change, against functional outcome measures in longitudinal studies. The preferred historical functional outcome measure in ophthalmology has been HCVA, but other measures, such as LCVA, colour vision, vision field and QoL, may matter as much, if not more, to patients in their daily lives. The FDA and EMA will not accept a retinal OCT parameter as a trial endpoint in and of itself, without such validation, and novel outcome measures will need to bwe used in two randomised controlled trials before they are likely to gain acceptance for licensing claims.

In turn, the regulatory authorities must embrace the multidimensional nature of visual function, which encompasses so much more than just HCVA. In using the readily measurable distance HCVA as the sole proxy for the measurement of vision impairment for decades, the vision community has perhaps scored its own goal. But the time has come to embed a more comprehensive appraisal of vision function in clinical trial design. The International Classification of Disease V.11 (https:/icd.who.int/), due for formal release soon, provides a more nuanced classification of vision function, which may better demonstrate the efficacy of interventions.

In ophthalmology, there is currently no PROM that is accepted by the FDA. The National Eye Institute Visual Function Questionnaire (NEI-VFQ) and its neuromodule were not developed or validated in a way that adheres to the FDA's guidance on PROM development ${ }^{43}$ and will never be acceptable for medical product development to support labelling claims. For QoL to take a more central stage in clinical trials, psychometrically validated PROMs will be required.

\section{PATIENT INVOLVEMENT}

It is now widely accepted that patients and their advocates must be involved in every phase, from the conception of a trial idea through to its design, analysis, publication and strategies for the dissemination of findings internationally. They also have an important role to play in the approval process with regulatory authorities.

There has been more active involvement of patients and patient representatives over the past few years in clarifying problem areas requiring improvement in the management of ON. Patient representatives have participated in major international neuro-ophthalmology meetings to provide a platform for public engagement (eg, https://www.uknosig.net/amsterdam2019report). Patient advocacy groups (European Patient Advisory Group) and patient organisations have been established and integrated into solid frameworks to support the delivery of advances in clinical practice for rare diseases and new research.

\section{EVIDENCE ON THE IMPACT OF ON ON QOL}

Despite the current challenge facing the inclusion of a QoL endpoint that captures the impacts of vision function in a clinical trial, the importance of finding a way forward is clear. ${ }^{44} 45$ Multiple studies, including our own large cohort $(n=267)$, report a statistical association between instruments measuring QoL (54-item Multiple Sclerosis Quality of Life Scale score, 39-item NEI-VFQ, NEI-VFQ-25 and the 10-item Neuro-Ophthalmic supplement) and OCT atrophy measures. ${ }^{45-49}$ Many studies also report an association between vision-related $\mathrm{QoL}$ and other measures of vision function. These studies have generally been limited by weak statistical analysis and the psychometric limitations of the available instruments. The North American Research Committee on Multiple Sclerosis reported survey data from 9107 patients, $66.7 \%$ of whom reported visual disability and $43.1 \%$ had previous ON. ${ }^{50}$ The MS Outcome Assessments Consortium reported that reduction in LCVA and vision-specific QoL persists for years after ON, even after HCVA recovery. ${ }^{15}$

Currently available generic tools that measure aspects (one or more dimensions) of vision-related QoL include the Raschvalidated Impact of Vision Impairment, which includes the ability to read and access information, mobility and emotional well-being. ${ }^{51} \mathrm{~A}$ frequently used instrument is the NEI-VFQ with neuro-ophthalmic module. ${ }^{52}$ These instruments have reasonable psychometric performance and yield measures suited to parametric statistical analysis but are not targeted to fully capture the impact of ON and its treatment on QoL. ${ }^{3153}$

For economic purposes, health-related QoL can readily be assessed using the EQ-5D-5L, which permits estimation of utility values associated with different health states. This instrument contains questions on mobility, self-care, usual activities, pain/ discomfort and anxiety/depression, scored 1-5, resulting in $3125\left(5^{5}\right)$ possible health states. Three new bolt-on items relate to vision, hearing and fatigue. ${ }^{54}$ The instrument also includes the EuroQL Visual Analogue Scale (EQ-VAS), which asks participants to rate their health on that day with a single number ranging from 0 to 100 (worst to best imaginable heath state). These tools permit estimation of utility coefficients and EQ-VAS scores, which are amenable to various statistical analysis approaches, such as ordinary least squares regression, with robust SE estimation..$^{55}$ By combining prevalence data and utility coefficients, the impact of loss of health resulting from an episode of acute ON can be estimated in terms of mean individual lifetime qualityadjusted life year loss.

\section{EARLY TREATMENT IS RELEVANT}

The failure of intravenous treatment with corticosteroids to improve vision outcomes in $\mathrm{ON}$ was reported by a recent Cochrane Systematic Review. ${ }^{56}$ However, common to all included studies was a long interval between onset of symptoms and initiation of treatment because inclusion criteria permitted up to an 8-day interval. ${ }^{3}$ In our clinical experience of treating high-risk patients with recurrent $\mathrm{ON}$, we have made two observations:

1. Loss of vision can be prevented if treatment is initiated in the early inflammatory phase, which patients recognise from previous attacks. ${ }^{5758}$

2. The critical time interval for treatment imitation is 48 hours. ${ }^{59}$

Hyperacute treatment versus no treatment or delayed treatment was associated with significant improvement in both functional (HCVA) and structural (OCT) outcome measures in a retrospective study on patients with relapsing $\mathrm{ON}$ and wellintegrated patient care pathways. ${ }^{59}$ To achieve this is clinically challenging. Importantly, we found that when the variable 'treatment initiation delay' was changed to more than 7 days, there was no beneficial effect on the outcome any more. ${ }^{5859}$

These observations are consistent with experimental evidence which supports the argument that there is a critical time window for treatment initiation in ON. Inflammation of the optic nerve precedes demyelination and axonal degeneration by about 2 days. ${ }^{60}$ Irreversible damage to the axonal cytoskeleton occurs within 5-7 days. ${ }^{39} 61$

A review of previous trials to explore the relationship between outcomes in ON, as well as the proposed critical window for corticosteroid treatment initiation, reveals a confused landscape: a recent, negative phase II trial in ON with amiloride permitted recruitment up to 28 days. ${ }^{62}$ There was an inclusion bias in the phase II erythropoietin ON treatment trial, with patients receiving the active drug on an average of 2 days earlier than 


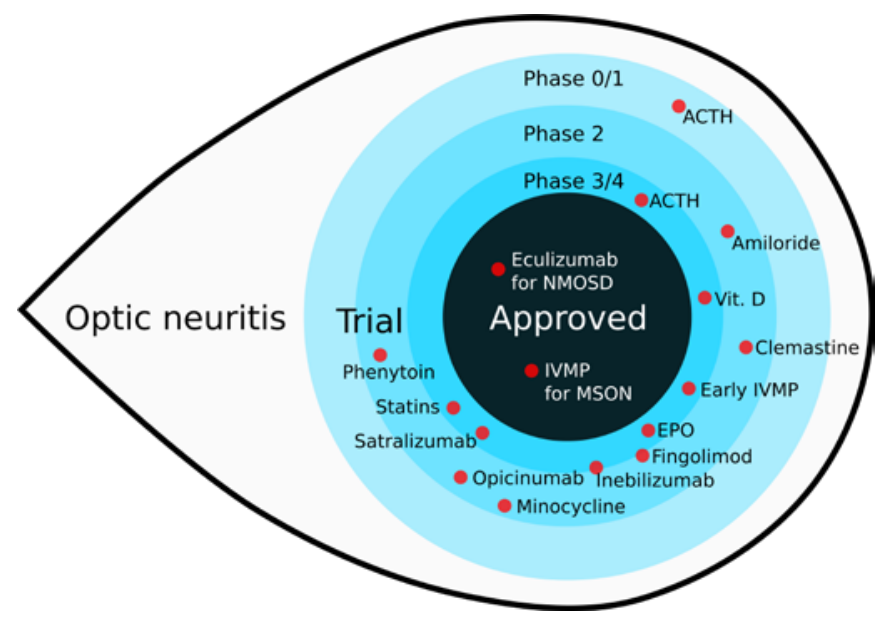

Figure 1 An overview of recent treatment trials in optic neuritis (updated from figure 1 in (25)). Eculizumab has been approved by the Food and Drug Administration for treatment of ON in neuromyelitis optica spectrum disease in July 2019. ON, optic neuritis.

the control group. ${ }^{63}$ The Simvastatin ON treatment trial groups differed in symptom onset to treatment initiation from 12 days for statins to 15 days for placebo, with neither primary nor secondary outcome measures achieving statistical significance. ${ }^{64}$ In the ON phenytoin trial, the average delay to treatment initiation was 8 days, ${ }^{65}$ with no improvement in vision but a significant reduction in retinal nerve fibre layer atrophy $(-7.15 \mu \mathrm{m}$, $\mathrm{p}=0.021){ }^{65}$

\section{RECRUITING RELAPSING ON}

In clinical practice, it is challenging to recruit patients with de novo ON within $<48$ hours of onset. Other than in stroke medicine, there are no patient pathways for making so quickly a reliable diagnosis of de novo ON. A practical way to circumvent this hurdle is to focus on patients with a known diagnosis of relapsing ON. ${ }^{59}$ The advantage here is that patients (1) are already known to the service, (2) have a baseline visual acuity and OCT, (3) have a diagnosis and (4) can be given a package of oral steroids at home. These patients will be aware of the cardinal symptoms of relapsing ON and can immediately start oral steroids and report straight back to the clinic for further investigations. This will explore the already proposed strategy of hyperacute treatment (prior to visual loss) in recurrent ON. ${ }^{57}$

\section{ECONOMIC COSTS}

New trials should consider collecting bottom-up cost and utilisation data from individual participants and their carers in order to permit cost-effectiveness analysis. Consensus guidelines for cost of vision loss studies have been developed by the Vancouver Economic Burden of Vision Loss Group. ${ }^{66}$ Important cost categories include direct monetary expenditure on healthcare (medical and other), monetary expenditure on non-healthcare items (such as low vision devices, refractive correction and travel costs), loss of family member productivity, loss of patient productivity and loss of patient well-being.

\section{RECOMMENDATIONS ON THE INCLUSION OF NOVEL PROGNOSTIC VARIABLES AND OUTCOME MEASURES IN FUTURE ONTTS}

The design of a new corticosteroid ONT trial should incorporate a quantitative marker to predict a treatment response. It should be predefined in the protocol that this marker will be used to stratify recruitment for preplanned subgroup analysis. The obvious candidate biomarker to this purpose are $\mathrm{Nf}$ proteins. $^{32} 33333867$ Additional baseline investigations should include the latest state-of-the-art immunological tests to aid ON classification, including anti-MOG, anti-AQP4 and anti-GFAP antibodies.

We feel that the most promising primary outcome measure for clinical trials is atrophy of the pRNFL ${ }^{6}$ and mGCIPL measured with OCT. ${ }^{21}$ Secondary functional outcome measures should still include HCVA (FDA and EMA requirements), $\mathrm{LCVA}^{15}$ and colour vision. ${ }^{16}$ Though the instruments are imperfect at present, the inclusion of QoL measures is also important and signals a direction of travel that patients rightly expect to see.

\section{TRIAL DESIGN FOR OTHER DRUGS}

The trial design aspects reviewed here specifically for corticosteroids also apply to other drugs. A review of recent trials in $\mathrm{ON}$ discussed these issues, and an overview of drugs of interest is shown in figure 1.

\section{CONCLUSION}

In this opinion piece, we have reviewed published evidence on the use of corticosteroids for the treatment of $\mathrm{ON}$. The one landmark trial that profoundly influenced medical management for decades was designed more than 30 years ago, in 1987. Revision of aspects of the trial design and outcome measures to align with contemporary clinical practice could shed vital new insights. We have proposed two key hypotheses for further investigation: that corticosteroid treatment has a profound effect on outcome in some cases of non-MSON and that the timing of treatment is critical, certainly for non-MSON and possibly for MSON. We hypothesise that swift intervention after the onset of ON could be sight-saving. In common with the findings from stroke trials, we feel this time window is likely to be in the order of 48 hours or even quicker. We further propose that HCVA is no longer the optimal primary outcome measure. Reasonable HCVA can be maintained despite poor visual function performance in other domains that greatly impact the daily lives of patients. A range of novel outcome measures have been validated which are sensitive, reproducible and more meaningful from a patient's perspective. It is vital to be able to predict treatment response. There have been too many failed trials that were followed by a range of post hoc hypotheses and analyses. Meaningful involvement of patients and advocates is an important aspect. There is a need to make this point to regulatory authorities. Likewise, incorporating a health economics perspective would potentially enhance health system sustainability. Taken together, we think there is enough evidence to make a case for a new corticosteroid treatment trial for acute ON.

\section{Author affiliations}

${ }^{1}$ Expertise Centrum Neuro-ophthalmology, Departments of Neurology \&

Ophthalmology, Amsterdam UMC, Amsterdam, The Netherlands

${ }^{2}$ Moorfields Eye Hospital, The National Hospital for Neurology and Neurosurgery,

UCL Institute of Neurology, London, UK

${ }^{3}$ Neuro-ophthalmology, Moorfields Eye Hospital and The National Hospital for Neurology \& Neurosurgery, London, UK

${ }^{4}$ Amsterdam UMC, VUMC Campus, Amsterdam, The Netherlands

${ }^{5}$ Neurology, VU University Medical Center, Amsterdam, The Netherlands

${ }^{6}$ Center of Neuroimmunology and Service of Neurology, Clinic Barcelona Hospital University, Barcelona, Spain

${ }^{7}$ ERN-EYE, Bordeaux, UK

${ }^{8}$ )Patient Organisation (Neuro-ophthalmology), Lisbon, Portugal

${ }^{9}$ Neuro-ophthalmologie Vereniging Nederland (KvK nummer 66260140), Amsterdam, Netherlands 
${ }^{10}$, National Hospital for Neurology and Neurosurgery, University College London Hospitals NHS Foundation Trust, St. Thomas Hospital, Moorfields Eye Hospital, London, UK

Correction notice This paper has been corrected since it appeared Online First. Figure 1 has been updated, sertalizumab and inebilizumab were phase $2 / 3$ trials and have been placed more clearly on the border. Author name 'Bob van Oosten' has been updated to 'Bob W van Oosten.

Twitter Tasanee Braithwaite @tasbraithwaite and Elena H Martinez-Lapiscina @ elenahlapiscina

Acknowledgements This research was supported by the National Institute for Health Research (NIHR) Biomedical Research Centre based at Moorfields Eye Hospital NHS Foundation Trust and UCL Institute of Ophthalmology. The views expressed are those of the authors and not necessarily those of the NHS, the NIHR or the Department of Health. We apologise to those authors whom we were not able to cite because of space limitations. EHM-L is employed by the European Medicines Agency (Human Medicines Evaluation) since 16 April 2019. This article is related with her activity affiliated to the Hospital Clinic of Barcelona and consequently, as external activity, it does not represent the views of the European Medicines Agency or its committees.

\section{Collaborators ERN-EYE.}

Contributors AP organised the UK NL Neuro-ophthalmology meeting in Amsterdam in March 2019, where the idea to this manuscript developed over a cup of coffee after the patient presentation session (https://uknos.com/ amsterdam2019report). He reviewed the literature and wrote the first draft.TB contributed many ideas at the Amsterdam meeting and cowrote the first series of drafts. BvO stimulated the discussion, encouraged the UK NL collaboration and revised the manuscript. LB substantially contributed to the body of research leading up to the meeting and manuscript, and provided statistical input and revised the manuscript. EHM-L is employed by the European Medical Agency and critically revised the manuscript, adding crucial references. RW was instrumental in implementing the patient session at the UK NL meeting, which was the result of many years of discussions with AP and ERN-EYE, who co-sponsored the meeting. He revised the manuscript. NW presented the patient perspective at the UK NL meeting which lay foundation to the core items integrated in this manuscript. He revised the manuscript. CW founded the neuro-ophthalmology patient organisation, where he is the acting president, and revised the manuscript. GTP pioneered many of the ideas which have made this manuscript possible and revised the manuscript.

Funding The authors have not declared a specific grant for this research from any funding agency in the public, commercial or not-for-profit sectors.

Competing interests None declared.

Patient consent for publication Not required.

Provenance and peer review Not commissioned; externally peer reviewed.

Open access This is an open access article distributed in accordance with the Creative Commons Attribution Non Commercial (CC BY-NC 4.0) license, which permits others to distribute, remix, adapt, build upon this work non-commercially, and license their derivative works on different terms, provided the original work is properly cited, appropriate credit is given, any changes made indicated, and the use is non-commercial. See: http://creativecommons.org/licenses/by-nc/4.0/.

ORCID iDs

Axel Petzold http://orcid.org/0000-0002-0344-9749

Elena H Martinez-Lapiscina http://orcid.org/0000-0003-4272-0826

\section{REFERENCES}

1 Whittam D, Wilson M, Hamid S, et al. What's new in neuromyelitis optica? a short review for the clinical neurologist. J Neurol 2017;264:2330-44.

2 Wynford-Thomas R, Jacob A, Tomassini V. Neurological update: MOG antibody disease. J Neurol 2019:266:1280-6.

3 Beck RW, Cleary PA, Anderson MM, et al. A randomized, controlled trial of corticosteroids in the treatment of acute optic neuritis. The optic neuritis Study Group N Engl J Med 1992;326:581-8.

4 Beck RW, Gal RL. Treatment of acute optic neuritis: a summary of findings from the optic neuritis treatment trial. Arch Ophthalmol 2008;126:994-5.

5 Cleary PA, Beck RW, Anderson MM, et al. Design, methods, and conduct of the optic neuritis treatment trial. Control Clin Trials 1993;14:123-42.

6 Petzold A, Wattjes MP, Costello F, et al. The investigation of acute optic neuritis: a review and proposed protocol. Nat Rev Neurol 2014;10:447-58.

7 Chen JJ, Tobin WO, Majed M, et al. Prevalence of myelin oligodendrocyte glycoprotein and Aquaporin-4-lgG in patients in the optic neuritis treatment trial. JAMA Ophthalmol 2018;136:419-22.

8 Moskopp D. Corticosteroids in the treatment of optic neuritis. N Eng/ J Med 1992;327:281; author reply 282
9 Achiron A, Djaldetti R, Ziv I. Corticosteroids in the treatment of optic neuritis. N Engl $\mathrm{J}$ Med 1992:327:281-2.

10 Beck RW. Oral versus intravenous corticosteroids in acute relapses of multiple sclerosis. Lancet 1997:349:1696.

11 Le Page E, Veillard D, Laplaud DA, et al. Oral versus intravenous high-dose methylprednisolone for treatment of relapses in patients with multiple sclerosis (COPOUSEP): a randomised, controlled, double-blind, non-inferiority trial. Lancet 2015:386:974-81.

12 Morrow SA, Fraser JA, Day C, et al. Effect of treating acute optic neuritis with bioequivalent oral vs intravenous corticosteroids: a randomized clinical trial. JAMA Neurol 2018;75:690.

13 Asgari N, Owens T, Frøkiaer J, et al. Neuromyelitis optica (NMO)--an autoimmune disease of the central nervous system (CNS). Acta Neurol Scand 2011;123:369-84.

14 Optic Neuritis Study Group. Visual function 15 years after optic neuritis: a final followup report from the optic neuritis treatment trial. Ophthalmology 2008;115:1079-82.

15 Balcer LJ, Raynowska J, Nolan R, et al. Validity of low-contrast letter acuity as a visual performance outcome measure for multiple sclerosis. Mult Scler 2017;23:734-47.

16 Barbur JL, Rodriguez-Carmona M. Colour vision requirements in visually demanding occupations. Br Med Bull 2017;122:51-77.

17 Almog Y, Gepstein R, Nemet AY. A simple computer program to quantify red desaturation in patients with optic neuritis. Graefes Arch Clin Exp Ophthalmol 2014:252:1305-8

18 Balcer LJ. Clinical trials to clinical use: using vision as a model for multiple sclerosis and beyond. J Neuroophthalmol 2014;34 Suppl:S18-23.

19 Balcer LJ, Miller DH, Reingold SC, et al. Vision and vision-related outcome measures in multiple sclerosis. Brain 2015;138:11-27.

20 Petzold A, de Boer JF, Schippling S, et al. Optical coherence tomography in multiple sclerosis: a systematic review and meta-analysis. Lancet Neurol 2010:9:921-32.

21 Petzold A, Balcer L, Calabresi PA, et al. Retinal layer segmentation in multiple sclerosis: a systematic review and meta-analysis. Lancet Neurol 2017:16:797-812.

22 Tewarie P, Balk L, Costello F, et al. The OSCAR-IB consensus criteria for retinal OCT quality assessment. PLoS One 2012;7:e34823.

23 Cruz-Herranz A, Balk LJ, Oberwahrenbrock T, et al. The APOSTEL recommendations for reporting quantitative optical coherence tomography studies. Neurology 2016;86:2303-9.

24 Schippling S, Balk LJ, Costello F, et al. Quality control for retinal OCT in multiple sclerosis: validation of the OSCAR-IB criteria. Mult Scler 2015:21:163-70.

25 Petzold A. Neuroprotection and visual function after optic neuritis. Curr Opin Neurol 2017:30:67-73

26 Nolan RC, Galetta SL, Frohman TC, et al. Optimal Intereye difference thresholds in retinal nerve fiber layer thickness for predicting a unilateral optic nerve lesion in multiple sclerosis. Journal of Neuro-Ophthalmology 2018;38:1-258

27 Coric D, Balk LJ, Uitdehaag BMJ, et al. Diagnostic accuracy of optical coherence tomography inter-eye percentage difference for optic neuritis in multiple sclerosis. Eur J Neurol 2017:24:1479-84.

28 Nolan RC, Liu M, Akhand O, et al. Optimal inter-eye difference thresholds by OCT in MS: an international study. Ann Neurol 2019:85:618-29.

29 Meneguette NS, de Carvalho JER, Petzold A. A 30 s test for quantitative assessment of a relative afferent pupillary defect (RAPD): the infrared pupillary asymmetry (IPA). J Neurol 2019;266:969-74.

30 Dean S, Mathers JM, Calvert M, et al. "The patient is speaking": discovering the patient voice in ophthalmology. Br J Ophthalmol 2017;101:700-8.

31 Braithwaite T, Calvert M, Gray A, et al. The use of patient-reported outcome research in modern ophthalmology: impact on clinical trials and routine clinical practice. Patient Relat Outcome Meas 2019;10:9-24.

32 Petzold A. Neurofilament phosphoforms: surrogate markers for axonal injury degeneration and loss. J Neurol Sci 2005;233:183-98.

33 Khalil M, Teunissen CE, Otto M, et al. Neurofilaments as biomarkers in neurological disorders. Nat Rev Neurol 2018;14:577-89.

34 Petzold A, Tisdall MM, Girbes AR, et al. In vivo monitoring of neuronal loss in traumatic brain injury: a microdialysis study. Brain 2011;134:464-83.

35 Petzold A, Keir G, Green AJE, et al. A specific ELISA for measuring neurofilament heavy chain phosphoforms. J Immunol Methods 2003;278:179-90.

36 Petzold A, Shaw G. Comparison of two ELISA methods for measuring levels of the phosphorylated neurofilament heavy chain. J Immunol Methods 2007;319:34-40.

37 Petzold A, Altintas A, Andreoni L, et al. Neurofilament ELISA validation. J Immunol Methods 2010;352:23-31.

38 Petzold A, Rejdak K, Plant GT. Axonal degeneration and inflammation in acute optic neuritis. J Neurol Neurosurg Psychiatry 2004;75:1178-80.

39 Bsteh G, Berek K, Hegen H, et al. Serum neurofilament levels correlate with retinal nerve fiber layer thinning in multiple sclerosis. Multiple Sclerosis Journal 2019;2011.

40 Jensen HA, Loukogeorgakis S, Yannopoulos F, et al. Remote ischemic preconditioning protects the brain against injury after hypothermic circulatory arrest. Circulation 2011;123:714-21.

41 Petzold A, Mondria T, Kuhle J, et al. Evidence for acute neurotoxicity after chemotherapy. Ann Neurol 2010;68:806-15 
42 Preische 0, Schultz SA, Apel A, et al. Serum neurofilament dynamics predicts neurodegeneration and clinical progression in presymptomatic Alzheimer's disease. Nat Med 2019:25:277-83.

43 FDA. Guidance for industry patient-reported outcome measures: use in medical product development 2009.

44 Ma S-L, Shea JA, Galetta SL, et al. Self-Reported visual dysfunction in multiple sclerosis: new data from the VFQ-25 and development of an MS-specific vision questionnaire. Am J Ophthalmol 2002;133:686-92.

45 Balk LJ, Coric D, Nij Bijvank JA, et al. Retinal atrophy in relation to visual functioning and vision-related quality of life in patients with multiple sclerosis. Mult Scler 2018;24:767-76.

46 Longbrake EE, Lancia S, Tutlam N, et al. Quantitative visual tests after poorly recovered optic neuritis due to multiple sclerosis. Mult Scler Relat Disord 2016;10:198-203.

47 Sabadia SB, Nolan RC, Galetta KM, et al. 20/40 or better visual acuity after optic neuritis: not as good as we once thought? J Neuroophthalmol 2016;36:369-76.

48 Garcia-Martin E, Ara JR, Martin J, et al. Retinal and optic nerve degeneration in patients with multiple sclerosis followed up for 5 years. Ophthalmology 2017;124:688-96.

49 Sanchez-Dalmau B, Martinez-Lapiscina EH, Pulido-Valdeolivas I, et al. Predictors of vision impairment in multiple sclerosis. PLoS One 2018;13:e0195856.

50 Salter AR, Tyry T, Vollmer T, et al. "Seeing" in NARCOMS: a look at vision-related quality of life in the NARCOMS registry. Mult Scler 2013;19:953-60.

51 Khadka J, Fenwick E, Lamoureux E, et al. Methods to develop the Eye-tem bank to measure ophthalmic quality of life. Optom Vis Sci 2016;93:1485-94.

52 Ramey NA, Butt Z, Burkat CN, et al. Patient-Reported outcomes: comprehensive analysis for the oculofacial clinician. Ophthalmic Plast Reconstr Surg 2014;30:279-89.

53 McAlinden C, Skiadaresi E, Moore J, et al. Subscale assessment of the NEI-RQL-42 questionnaire with Rasch analysis. Invest Ophthalmol Vis Sci 2011;52:5685-94.

54 Devlin NJ, Shah KK, Feng Y, et al. Valuing health-related quality of life: an EQ-5D-5L value set for England. Health Econ 2018;27:7-22.
55 Pullenayegum EM, Tarride J-E, Xie F, et al. Analysis of health utility data when some subjects attain the upper bound of 1 : are Tobit and CLAD models appropriate? Value Health 2010;13:487-94.

56 Gal RL, Vedula SS, Beck R. Corticosteroids for treating optic neuritis. Cochrane Database Syst Rev 2015.

57 Plant GT, Sibtain NA, Thomas D. Hyperacute corticosteroid treatment of optic neuritis at the onset of pain may prevent visual loss: a case series. Mult Scler Int 2011;2011:1-8.

58 Stiebel-Kalish $\mathrm{H}$, Hellmann MA, Mimouni M, et al. Does time equal vision in the acute treatment of a cohort of AQP4 and MOG optic neuritis? Neurol Neuroimmunol Neuroinflamm 2019;6:e572.

59 Osinga $E$, van Oosten $B$, de Vries-Knoppert $W$, et al. Time is vision in recurrent optic neuritis. Brain Res 2017;1673:95-101.

60 Shindler KS, Ventura E, Dutt M, et al. Inflammatory demyelination induces axonal injury and retinal ganglion cell apoptosis in experimental optic neuritis. Exp Eye Res 2008;87:208-13.

61 Zhu B, Moore GR, Zwimpfer TJ, et al. Axonal cytoskeleton changes in experimental optic neuritis. Brain Res 1999;824:204-17.

62 McKee JB, Elston J, Evangelou N, et al. Amiloride clinical trial in optic neuritis (action) protocol: a randomised, double blind, placebo controlled trial. BMJ Open 2015;5:e009200.

63 Sühs K-W, Hein K, Sättler MB, et al. A randomized, double-blind, phase 2 study of erythropoietin in optic neuritis. Ann Neurol 2012;72:199-210.

64 Tsakiri A, Kallenbach K, Fuglø D, et al. Simvastatin improves final visual outcome in acute optic neuritis: a randomized study. Mult Scler 2012;18:72-81.

65 Raftopoulos R, Hickman SJ, Toosy A, et al. Phenytoin for neuroprotection in patients with acute optic neuritis: a randomised, placebo-controlled, phase 2 trial. Lancet Neurol 2016;15:259-69.

66 Frick KD, Kymes SM, Lee PP, et al. The cost of visual impairment: purposes, perspectives, and guidance. Invest Ophthalmol Vis Sci 2010;51:1801-5.

67 Barro C, Benkert P, Disanto G, et al. Serum neurofilament as a predictor of disease worsening and brain and spinal cord atrophy in multiple sclerosis. Brain 2018;141:2382-91. 